\title{
Galileo and DSM
}

\author{
Alen J. Salerian* \\ The Salerian Center for Neuroscience and Pain, Washington, DC, USA
}

There is a passage in Galileo's Dialogue ${ }^{1}$ in which Salvioti, Galileo's fictional champion of the Copernican theory, says:

We are certain that the earth has a center, toward which we see that all its parts move. We understand that as they move toward the center of the earth, they move toward their universal mother. Now, let us have the grace to abandon the argument that their natural instinct is to go not toward the center of the earth, but toward the center of the universe.

Salvioti's insight- that the earth is not the center of the universe-which clashed with 2000 years of Ptolemaic assumptions, is a useful metaphor as we ponder the predominant influence of the Diagnostic and Statistical Manual of Mental Disorders (DSM) ${ }^{2}$ on neuroscience.

Since the birth of DSM half a century agoa significant step forward in legitimizing psychiatrynew insights in the genesis of human behavior (region-specific brain function, neurotransmitters, and thermodynamic laws) have emerged. Today, for instance, there is recognition that the amygdala plays a key role in our fear responses, the prefrontal cortex mediates mood and executive function, and the thalamus is a crucial filter for sensory input. ${ }^{3}$ Molecular advances have also provided a framework of knowledge about key neurotransmitters and neuromodulators, such as brain-derived neurotropic factor, G protein, CAMP, and others. ${ }^{4}$

In contrast, concepts regarding complexity, butterfly effect, and eigenvalues, by and large, have received sparse attention in the neurosciences.

Richard D. Feynman's observation, "Nature is absurd from the point of view of common sense," offers a glimpse into the mismatch between DSM and neuroscientific advances. ${ }^{5}$ Due to our sensory limitations, nature may seem absurd. There are many influences that are unobservable by our sensory system but that can be explained through eigenvalues ${ }^{6}$ such as gravity, sound waves, or vibration.

The theories of Poincare and Lorentz ${ }^{7}$ propose that complex systems (e.g., brain function) are vulnerable

\footnotetext{
*Address for correspondence: Alen J. Salerian, MD, The Salerian Center for Neuroscience and Pain, 5028 Wisconsin Ave., NW, Suite 220, Washington, DC 20016, USA

(Email: alensalerian@yahoo.com)
}

to initial errors. This phenomenon, which is recognized as the butterfly effect, suggests that an early diagnostic error may lead to delayed and magnified adverse outcome.

It is plausible that a major DSM flaw-to classify "disease," "disease complication," or "disease progression" as independent "comorbid disorders" — has inhibited a pathophysiologically sound pursuit of accurate diagnosis. It is also plausible that various DSM disorders are artifacts or complications of not yet recognized brain dysfunctions. For instance, addictive disorders, depression, and schizophrenia may represent "disease complications."

Two independent observations seem to support the possibility that addictive disorders are complications of brain dysfunction:

1. f-MRI-based findings are consistent with abnormalities associated with the propensity for addictive behavior. $^{8}$

2. Logical behavior, as the domain of prefrontal cortex function, leads to the conclusion that recurrent selfharm by voluntary intake of a harmful substance can only occur in the absence of a robust and functional prefrontal cortex. ${ }^{3}$

Chronic schizophrenia may represent the end stage of progressive degeneration similar to neurosyphilitic dementia caused by Treponema pallidum infections. ${ }^{9}$ Treponema pallidum dementia has been associated in MRI studies with atrophy of the frontal and temporal neural cortex and positive CSF titers of Treponema pallidum with signs and symptoms of neurosyphilitic psychosis. It may be possible that a brain dysfunction of diverse origins may progress to cause brain injuries with a cluster of symptoms corresponding to regional brain dysfunction. In general, a psychotic episode precedes negative symptoms, and effective treatment prevents worsening of psychosis. ${ }^{10}$ It is also true that chronic schizophrenia is devoid of a predominant genetic marker. 9 Collectively, all of the above are consistent with the hypothesis that chronic schizophrenia may represent the end-stage of a psychotic disorder.

Evidence suggests that an overactive amygdala is a biological marker for some depressions, which is consistent with the influence of amygdala activity on fear and stress and data from the unpredictable chronic mild stress (UCMS) rodent model. Of essence 
are strong, statistically significant correlations between depression in humans with its reversal by antidepressants and the response to antidepressants in UCMS. ${ }^{11,12}$

Let us consider a hypothetical clinical challenge of someone with comorbid conditions of alcohol abuse, social phobia, and generalized anxiety disorder. In general, to address alcohol abuse, a cluster of therapeutic interventions (group therapy, AA, abstinence, and avoidance of benzodiazepines) may be offered, although they may hinder effective biological or behavioral treatment for social phobia and generalized anxiety disorder. In essence, treating alcohol abuse may compromise the appropriate treatment for social phobia and generalized anxiety disorder. With a diagnostic system rooted in neurophysiology, neuroanatomy, and brain complexity, it may be possible to view generalized anxiety disorder and social phobia as related to amygdala dysfunction (greater sensitivity to external threats) and also view alcohol abuse as correlated with diminished prefrontal cortex function (poor impulse control).

The above examples are consistent with the limitations of a linear system to measure the dynamic complexity of brain dysfunction and the advantages of a medically sound conceptualization of neuropsychiatric disorders. DSM's insensitivity to complexity is of essence. Complexity-now the backbone of modern neuroscience-applies to everything in neuroscience, to schizophrenia as well as to depression, unlike DSM and its rigid compartmentalization of dynamic brain function and human behavior.

It is also true that our current regulatory guidelines demand strict adherence to DSM and thus may inadvertently contribute to inaccurate collection of data, which compromises future research dependent on such data. For instance, searching for solutions for chronic schizophrenia without entertaining the possibility that it may be a complication of an untreated psychotic condition may be as wise as treating diabetic retinopathy without realizing its origin.

In conclusion, statistically meaningful correlations support the observation that the mismatch between inherent DSM deficits (insensitivity to eigenvalue and butterfly effect and the relative absence of pathophysiology) and the essential laws of brain function (dynamic complexity and region-specific function) have possibly contributed to the generation of phantom disorders and invalid research and treatment.

Neuroscientific advances may require a diagnostic system that incorporates knowledge of regional brain function, thermodynamics, complexity, and eigenvalue problems in addition to behavioral and molecular data. For that to happen, our nomenclature should reflect appropriate labels, i.e., amygdala hyperfunction, prefrontal cortex dysfunction, hypothalamic hyperactivity, etc.

The shortcomings of the DSM pose serious impediments to research and treatment. An urgent reassessment of DSM would enhance neuroscientific progress.

\section{References}

1. Galilei G. Le opera di Galileo Galilei. Favaro A, ed. 20 vols. Florence: Barbera; 1929-1939. Abbreviated OGG.

2. American Psychiatric Association. Diagnostic and Statistical Manual of Psychiatric Disorders. Washington, DC: American Psychiatric Association; 2004.

3. Salerian AJ, Altar CA. The prefrontal cortex influence over subcortical and limbic regions governs antidepressant response by $\mathrm{N}=\mathrm{H} /(\mathrm{M}+\mathrm{R})$. Psychiatry Res. Neuroimaging. 2012. In press.

4. Feyman, Richard. The Character of Physical Law. MIT Press. 1967. Library of Congress Catalog Card \#6714527. ISBN 0-679-60127-9.

5. Nolte J. The Human Brain: An Introduction to Its Functional Anatomy. Philadelphia: Mosby Elsevier; 2008.

6. Microsoft Encarta College Dictionary. New York: St. Martin's Press; 2001.

7. Mitchell M. Complexity. New York: Oxford University Press; 2009.

8. Volkow ND, Fowler JS. Addiction, a disease of compulsion and drive: involvement of the orbitofrontal cortex. Cereb Cortex. 2000; 10(3): 318-325.

9. Kayal AK, Goswami M, Das M, Paul B. Clinical spectrum of neurosyphilis in North East India. Neurol India. 2011; 59: 344-350.

10. Patel NH, Vyas NS, Puri BK, et al. A. Positron emission tomography and schizophrenia: a new perspective. J Nucl Med. 2010; 51(4): 511-520.

11. Willner P. Chronic mild stress (CMS) revisited: consistency and behavioral neurobiological concordance and the effects of CMS. Neuropsychobiology. 2005; 52: 90-110.

12. Sibille E, Wang $\mathrm{Y}$, Joeyen-Waldorf J, et al. A molecular signature of depression in the amygdala. Am J Psychiatry. 2009; 166: 1011-1024. 